\title{
Quantitative analysis of the thermal distortions in a 100 W CW Nd:YAG ceramic slab laser
}

\author{
A. Lapucci \\ antonio.lapucci@inoa.it \\ M. Ciofini \\ E. Favilla \\ E. Sani \\ M. De Rosa \\ G. Mincuzzi
}

\section{A. Rocco}

\begin{abstract}
Istituto Nazionale di Ottica Applica INOA-C.N.R. Largo E.Fermi 6 I-50125, Firenze - Italy
\end{abstract}
Istituto Nazionale di Ottica Applica INOA-C.N.R. Largo E.Fermi 6 I-50125, Firenze - Italy

Istituto Nazionale di Ottica Applica INOA-C.N.R. Largo E.Fermi 6 I-50125, Firenze - Italy

Istituto Nazionale di Ottica Applica INOA-C.N.R. Largo E.Fermi 6 I-50125, Firenze - Italy

Istituto Nazionale di Ottica Applica INOA-C.N.R. Via Campi Flegrei 3480078 Napoli - Italy

Istituto Nazionale di Ottica Applica INOA-C.N.R. Via Campi Flegrei 3480078 Napoli - Italy

Istituto Nazionale di Ottica Applica INOA-C.N.R. Via Campi Flegrei 3480078 Napoli - Italy

Making use of an interferometric diagnostic system, we analyze the thermo-mechanical distortions taking place in the slab shaped ceramic $\mathrm{Nd}$ :YAG active medium of a $100 \mathrm{~W}$ class laser. These distortions are collected in different pumping regimes, both in static situations and during transient warm-up, and compared to the results of computer simulations. This procedure enables us to determine the relevance of different stress causes and thus to increase the specific power extraction of the active slab module. [D0I: 10.2971/je0s.2007.07020]

Keywords: diode pumped solid state lasers, ceramic YAG lasers, slab lasers

\section{INTRODUCTION}

In the recent past we operated a Nd:YAG ceramic zig-zag slab laser face pumped by two horizontal stacks of passively cooled QCW laser diode bars with total pumping power up to $1200 \mathrm{~W}$ [1]. The pump radiation coupling was investigated and optimized resulting in a maximum slope efficiency as high as $51 \%$ [2]. The maximum extracted power, averaged over the $150 \mu$ s pulse duration, reached $350 \mathrm{~W} @$ a 15\% duty cycle. Different optical cavity schemes have also been adopted on this device and the optical quality factor of the extracted beam was thoroughly characterized [3] as a function of different pumping power levels.

In this paper we report on the experiments performed on the same ceramic YAG slab pumped by two vertical stacks of actively cooled CW diode bars. Given the smaller pumped volume and the higher average thermal loading of the YAG slab, the optical output of the laser resulted not stable above a certain pumping power density (of the order of $750 \mathrm{~W} / \mathrm{cm}^{3}$ ). For this reason we inserted the active medium complete of the pumping units in one arm of a Mach-Zehnder interferometer with the aim of optically characterize the YAG slab deformations induced by the pump radiation loading. These distortions have been analyzed, in terms of aberration polynomial coefficients, in both static and transient experiments. The extracted wavefront data in different pumping conditions enabled us to establish a hierarchy among the various distortions and among their possible causes. These causes have also been verified through Finite Element Method (FEM) thermomechanical computer simulations. The knowledge of the thermal induced wavefront distortions turns out to be fundamental for the design and operation of high power solid-state lasers and particularly of slab lasers. Thus they have been widely investigated in the past [4]-[6]. These wavefront distortions may have different origin, such as transverse variation of the refractive index due to thermal gradients within the active medium, or variations caused by stress-optical effects or, finally, beam distortions induced by physical deformation of the relevant faces of the lasing medium. Obviously a correct interpretation of the causes of wavefront distortions can eventually lead to designs that correct these distortions or at least that result less sensitive to their occurrence [7]-[9].

\section{LASER OUTPUT POWER AND TEMPORAL STABILITY}

The optical pumping scheme of our CW ceramic slab laser is similar to that already reported in our previous papers [1][3]. The polycristalline ceramic YAG slab is the one adopted in the QCW operation experiments [1]-[3]. It has $10 \mathrm{~mm} \times$ $3 \mathrm{~mm}$ cross-sectional dimensions, it is doped with a $1 \%(\mathrm{wt})$ of Neodimum and it was produced by Konoshima Chemicals (Baikowski Group). The slab has Brewster-angle-cut surfaces (with an edge angle $\alpha=90^{\circ}-\theta_{B}=28.86^{\circ}$ ) and is $114.3 \mathrm{~mm}$ 
(edge-to-edge) long. In this new configuration the slab is face pumped by two vertical stacks of actively cooled CW diode bars (emitting at $808 \mathrm{~nm} \pm 3 \mathrm{~nm} @ 20^{\circ} \mathrm{C}$ ) capable to deliver more than $600 \mathrm{~W}$ each at a driving current of $70 \mathrm{~A}$. The laserdiode stacks are produced by Nuvonyx Inc. (Bridgeton, MO), and are based on ten $60 \mathrm{~W}$ bars with a $1.6 \mathrm{~mm}$ pitch. They are set $12 \mathrm{~mm}$ far from the slab with the Fast Axis (FA) parallel to the slab elongation direction. Having a $38^{\circ}$ FA divergence they produce an illumination area of $10 \mathrm{~mm} \times 24 \mathrm{~mm}$ on the slab faces. This corresponds, in case of equal pump power, to an irradiance twice as high as that adopted in the QCW experiments.

The resonator adopted for the experiments described in this paper, is a classical stable resonator. Mirrors are a concave spherical rear mirror (High reflectivity [> 99.9\%] multilayer dielectric coated, $1 \mathrm{~m}$ R.O.C.) and a flat output coupler (dielectric coating with $R=85.0 \%$ at $1.064 \mu \mathrm{m}$ ).

Figure 1 shows the power extraction characteristic of the CW laser configuration vs. the optical pump power. The dashed area indicates pumping levels producing power extractions that change abruptly in the course of time. The extracted power reaches its maximum level after several seconds of warm up and then is progressively reduced.

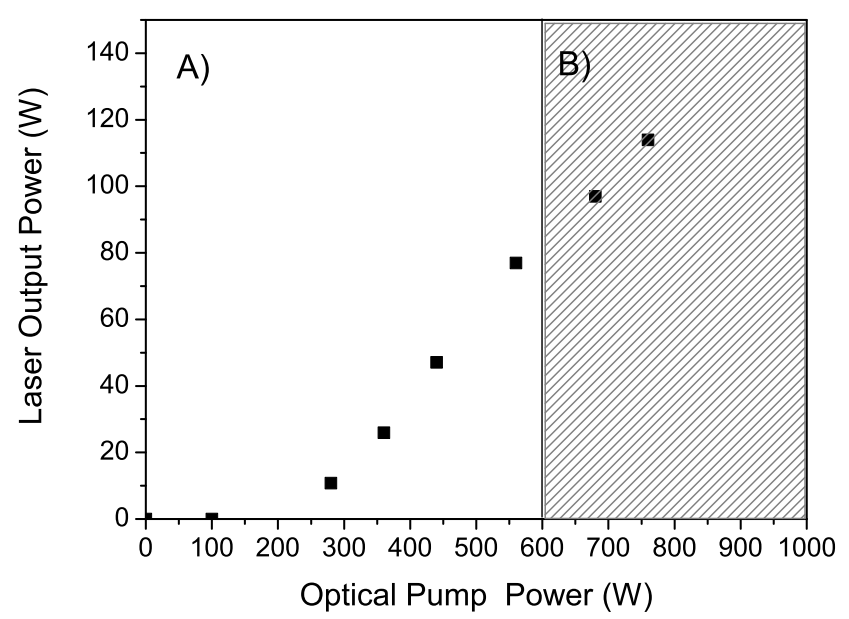

FIG. 1 Laser power extraction data. The dashed area corresponds to pumping powers that do not allow a stable extraction of the maximum optical power.

This behavior is shown in Figure 2 for several different diode currents, that means different optical powers impinging on the slab pumped section.

After stabilization on a lower output power level, a higher extraction can be restored correcting the resonator alignment in the transverse direction corresponding to the thin size of the slab. This happens even though the resonator is set, before every run, in a condition of best alignment, in the sense of maximum power extraction for low pumping powers or for 'cold' active medium. In order to investigate the possible causes of this thermal behavior we inserted the laser gain module in a diagnostic interferometric set-up. The laser gain module consists in the ceramic YAG slab, with its mounting and cooling

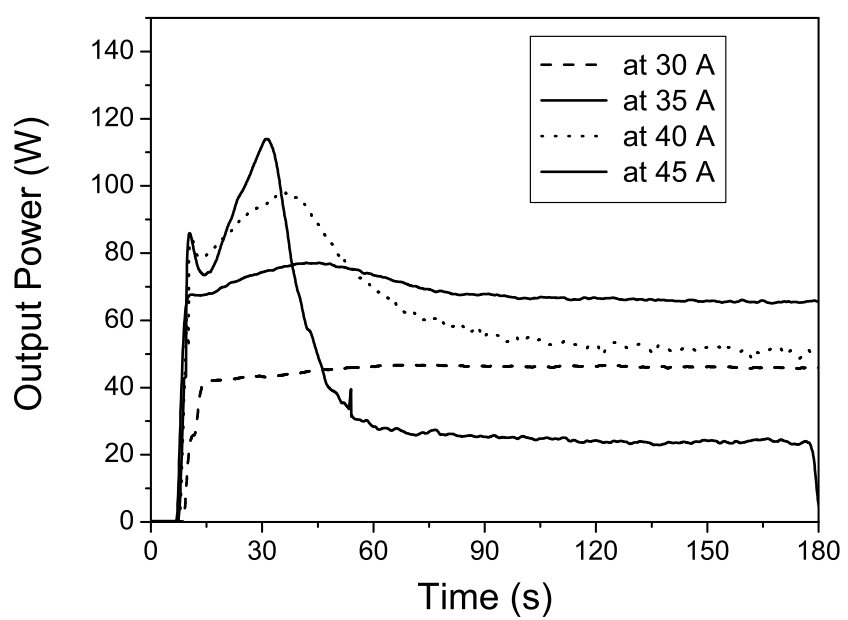

FIG. 2 Temporal evolution of the extracted power in the first few minutes of operation, for different pumping diode currents corresponding to the last four points of Figure 1

system, and in the pumping units, based on the diode bar stacks and their cooling systems.

\section{INTEFEROMETRIC MEASUREMENTS OF THE SLAB DEFORMATIONS}

The diagnostic setup is a Mach-Zehnder interferometer with a $5 \mathrm{~mW}$ HeNe laser (Figure 3).

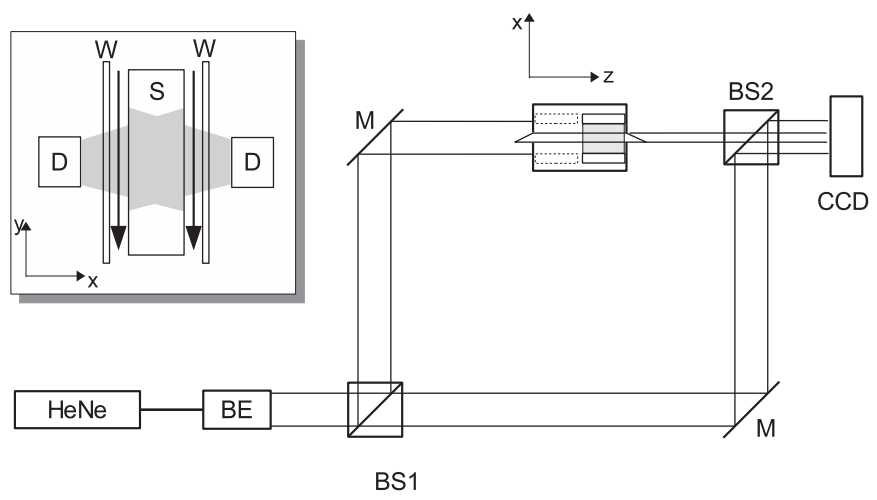

FIG. 3 Schematic view of the slab laser gain module inserted in the Mach-Zehnder interferometer. BE: beam expander; BS: beam splitter; M: mirror; CCD: coupled charge device camera. The inset shows the cross-sectional view of the slab (S) pumped by two diode laser arrays (D) through sapphire windows (W) and a cooling water flow (symbolized by the two vertical black arrows).

The laser beam is expanded to about $40 \mathrm{~mm}$ diameter by a telescope and accurately collimated. A first beam splitter BS1 divides the beam into two parts. The transmitted part (reference beam) propagates in air and is sent to a second beam splitter BS2. Reflected light from BS1 (object beam) is sent through the ceramic slab and finally on BS2, where it interferes with the reference beam. The light follows a zig-zag optical path inside the slab, very similar to that of the laser beam obtained when the slab is inserted in the optical resonator. At one output port of BS2 a CCD camera records the interference pattern. The CCD camera has an active area made by a $1600 \times 1200,2 \mathrm{D}$-array of $7 \mu \mathrm{m}$-size pixels (interpixel area in- 
cluded). The fringe pattern corresponding to the section of the slab has an effective dimension of $270 \times 910$ pixels. An interferential filter for $633 \mathrm{~nm}$ is placed in front of the camera, in order to avoid background radiation, especially that coming from the pump diodes around $808 \mathrm{~nm}$, and from the optical de-excitation of the pumped slab at $1064 \mathrm{~nm}$, both scattered through the slab aperture. The two interfering beams are firstly aligned along the same axis, after BS2, then the reference beam is slightly tilted with respect to the object one, thus a pattern of fringes occurs. Assuming the two interfering wavefronts being plane waves, the fringes are equally spaced and perpendicular at the plane determined by the tilt angle. When the ceramic slab is pumped by the diode arrays, non-radiative de-excitation induces thermal deformations. As a result the incoming object wavefront changes after passing through the slab, thus changing the initial fringe pattern. Two typical fringe patterns acquired by the CCD camera are shown in Figure 4 corresponding to (a) a cold situation, i.e. with diode radiation off, and (b) to a hot situation, with diode radiation on. (a)

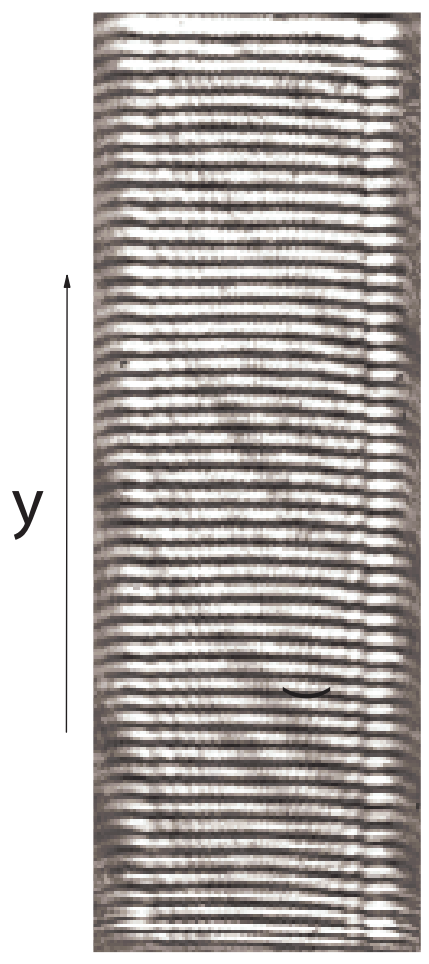

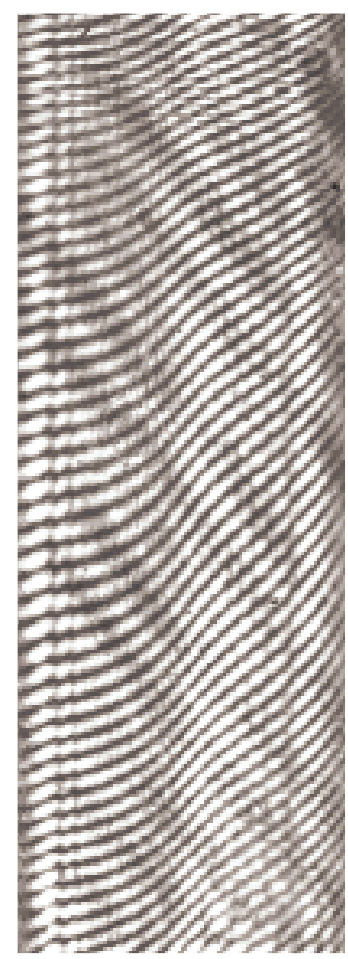

(b)
FIC. 4 Fringe structure obtained from the Mach-Zehnder interferometer in the case of (a) diodes off and (b) diodes on (45 A current)

To retrieve the information on the wavefront coming out from the pumped slab, each row along the $y$ direction of the recorded interferogram is numerically Fourier transformed and filtered, in order to remove noise and unavoidable spatial modulation of intensity. Then, the filtered spectrum is shifted and inversely Fourier transformed to obtain the phase information, wrapped around $2 \pi$. Finally, after processing of all the rows, a two-dimensional unwrapping algorithm produces a 2-d continuous map of the complete phase distribution. The initial phase map (with pump diode turned off) is subtracted to successive phase maps, so that only the differential phase change $\Delta \Phi(x, y)$ due to the slab deformations is retained. Thus small imperfections of the initial wavefront can be neglected. The interpretation of the resulting wavefront is performed with reference to a set of standard aberration components. We choose to model the wavefront as a linear combination of eight terms, accounting for spherical aberration, tangential coma, sagittal coma, astigmatism, defocusing, $x$-tilt, $y$ tilt and piston, respectively [10]:

$$
\begin{aligned}
\Delta \Phi(x, y) & =A\left(x^{2}+y^{2}\right)^{2}+B x\left(x^{2}+y^{2}\right)+C y\left(x^{2}+y^{2}\right)+ \\
& +D\left(x^{2}+3 y^{2}\right)+E\left(x^{2}+y^{2}\right)+F y+G x+H
\end{aligned}
$$

The polynomial expression of Eq. 1 is fitted to experimental wavefronts $\Delta \Phi(x, y)$ with $A$ to $H$ as free fit parameters, according to a linear least-square fitting procedure. Figure 5 shows (a) an example of an experimental wavefront extracted from the recorded interferogram, (b) the final result of a fitting procedure, and (c) the residual between them.
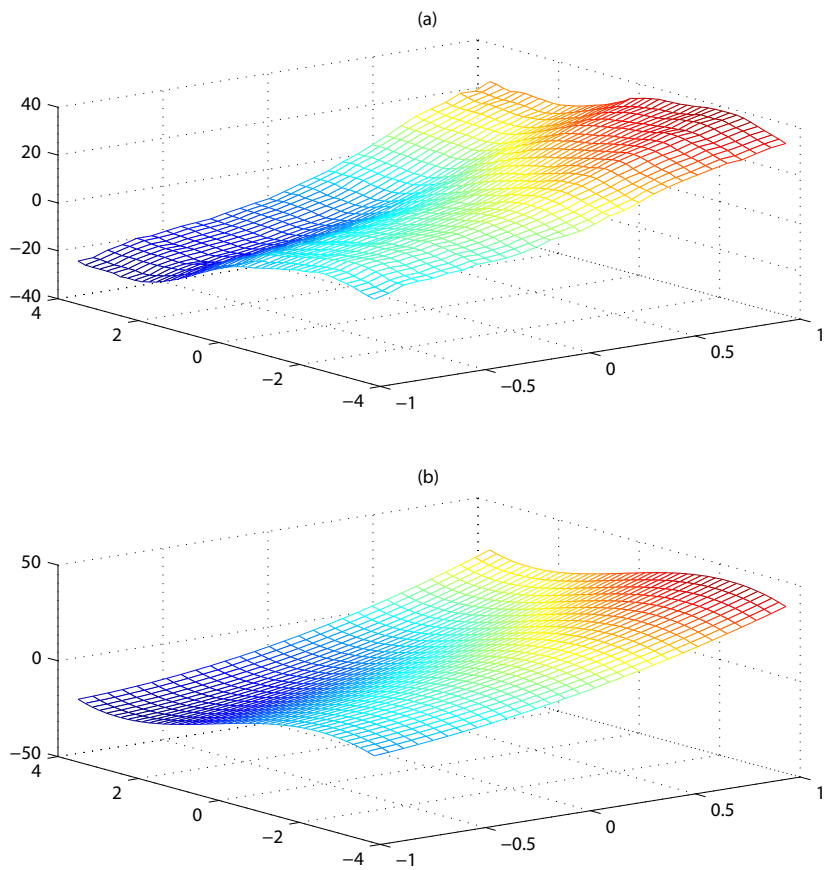

(c)

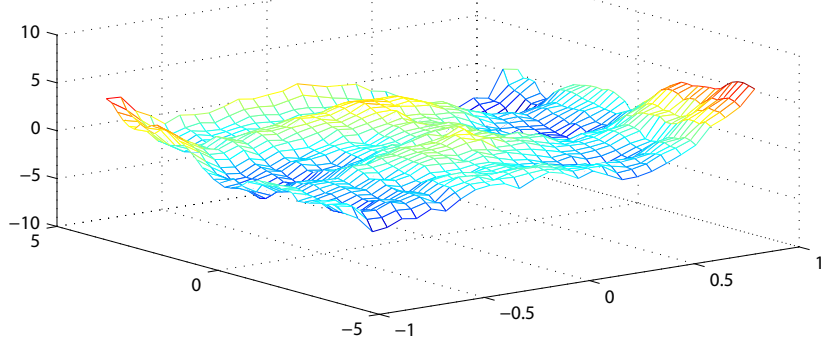

FIG. 5 (a) Experimental phase map of the wavefront distorted by passing through the symmetrically pumped slab with $45 \mathrm{~A}$ of diode current. (b) Best fit of the experimental map according to Eq. 1. (c) Residual difference between experimental data and fitted function.

This choice of the basis to expand the experimental wavefronts is not unique and one could choose other sets of aber- 
ration functions, such as Zernike polynomials. The set chosen, however, turns out to be capable of including all the relevant features. This fact is also confirmed in Figure 5 by the limited residuals between experimental wavefronts and their best fit. In Figure 6 typical values of aberration coefficients are reported, obtained in a standard pumping configuration, i.e. with both stacks driven by the same current.
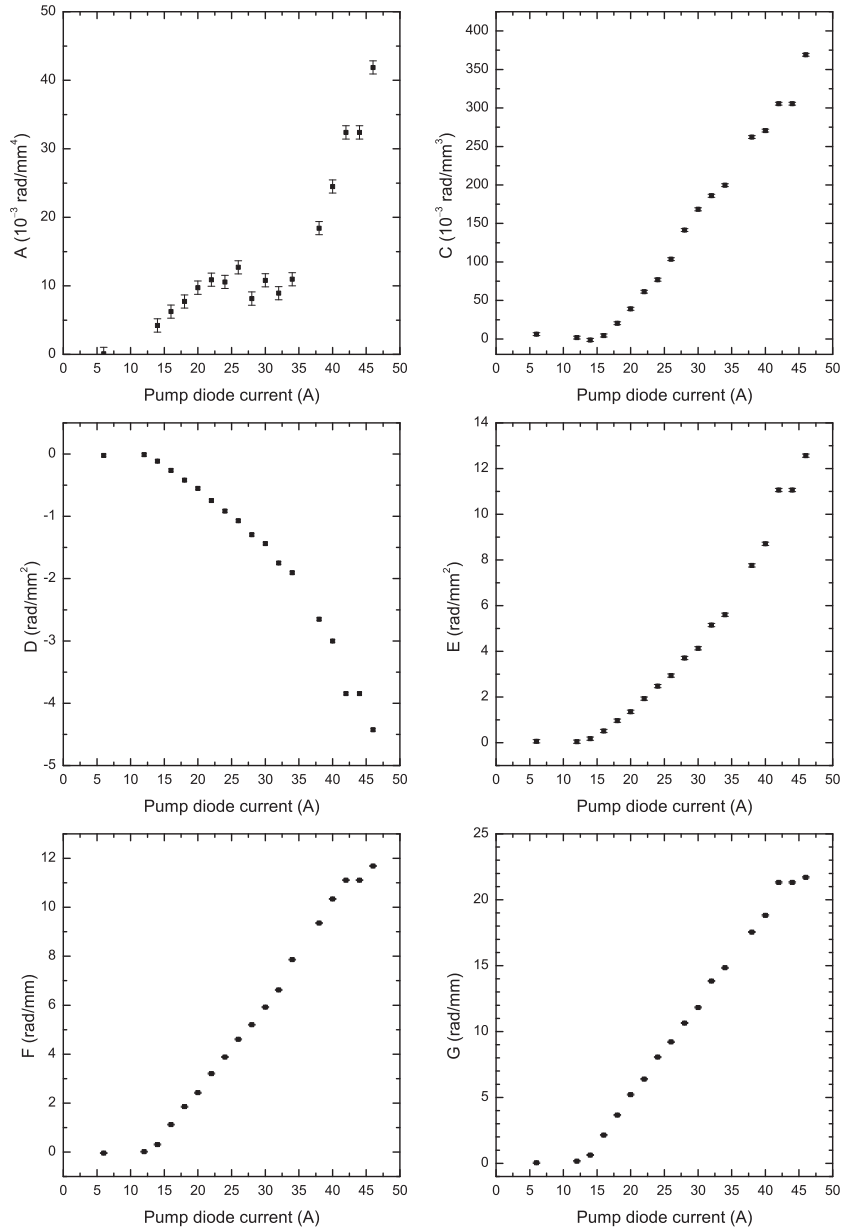

FIG. 6 Relevant aberration coefficients as a function of the pump diode current, in case of symmetric pumping.

We omitted the plot of the $B$ coefficient as it produces negligible contributions to the phase distribution. Indeed considering the $x$ and $y$ apertures determined by the rectangular crosssection of our slab we can estimate the maximum Optical Path Difference $\left(\mathrm{OPD}=\Delta \Phi_{\max } \lambda / 2 \pi\right)$ of each aberration as:

$20 \lambda$ for $y$ tilt $[F y]$;

$10 \lambda$ for $x$-tilt $\quad[G x]$

$7 \lambda$ for sagittal coma $\left[C y\left(x^{2}+y^{2}\right)\right]$;

$5 \lambda$ for spherical aberration $\left[A\left(x^{2}+y^{2}\right)^{2}\right]$;

$3 \lambda$ for $x$-defocusing $\left[(D+E) x^{2}\right]$;

$2 \lambda$ for $y$-defocusing $\left[(3 D+E) y^{2}\right]$;

$0.1 \lambda$ for tangential coma $\left[B x\left(x^{2}+y^{2}\right)\right]$.

Figure 6 clearly shows aberrations to grow with increasing driving current, that means with the slab thermal loading. Using the relation

$$
\text { R.O.C. }=a^{2} / 2 \mathrm{OPD}
$$

where R.O.C. is the wavefront Radius Of Curvature, a is the wavefront semi-aperture, and OPD is the optical path differ- ence between axial and aperture constant phase surfaces, we can calculate from $x$ and $y$ defocusing terms the equivalent thermal lens in the two transverse directions as:

$$
\begin{aligned}
& \left(f_{\mathrm{th}}\right)_{x}=\frac{\pi}{\lambda(D+E)} \\
& \left(f_{\mathrm{th}}\right)_{y}=\frac{\pi}{\lambda(3 D+E)}
\end{aligned}
$$

From Eq. (4) we obtain extremely large focal length values for the slab large transverse cross-sectional direction, decreasing down to 5 meters at the maximum adopted current level (50 A). This confirms the negligible thermal gradients produced by our geometry in the $y$ direction. On the contrary, thermal gradients in the $x$ direction have a more sizeable effect on the focal length in that direction, which is lower than $1 \mathrm{~m}$ for pump currents greater than $\sim 35 \mathrm{~A}$. Tilt contributions in both transverse directions and thermal lens in the slab thin crosssection are summarized in Figure 7. The same tests have been performed in cases when only one array was operated.
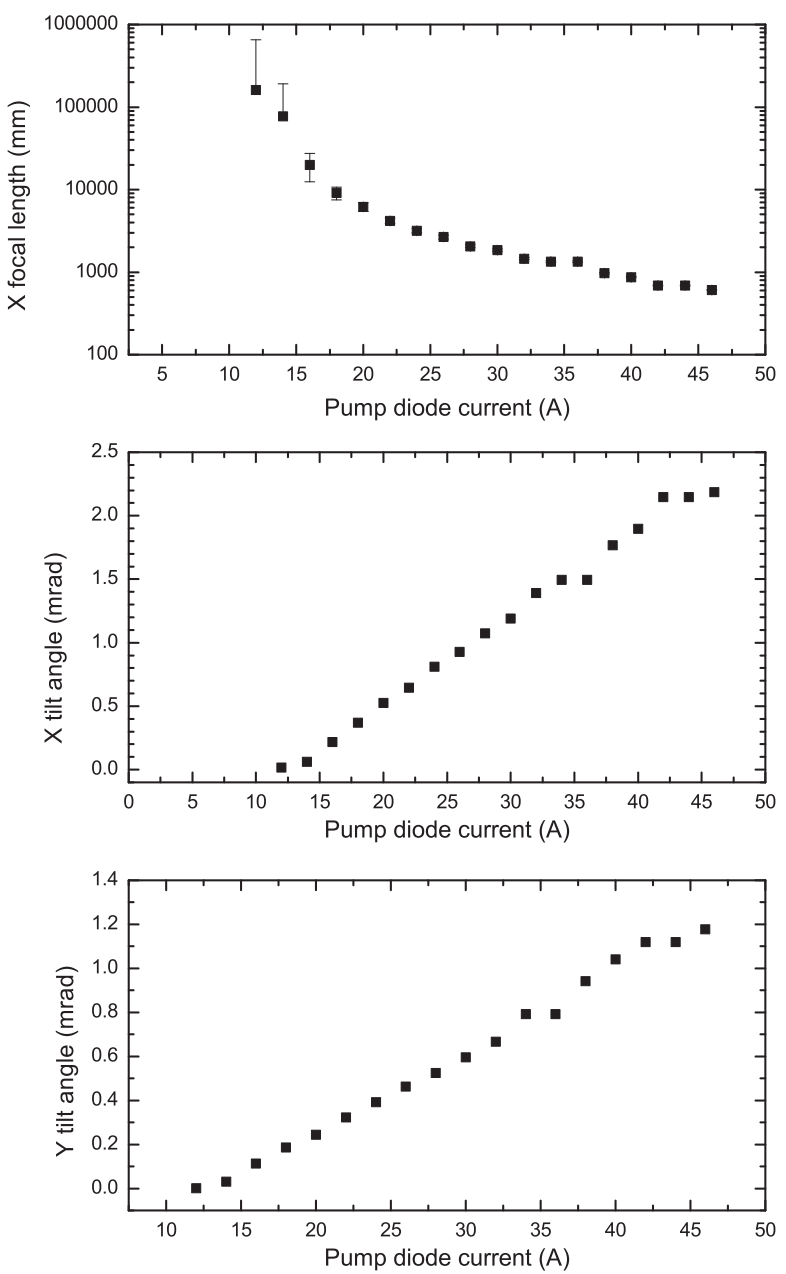

FIG. 7 Focal length in the $x$ direction and tilt angle in both transverse directions, as a function of pump diode current.

Figure 8 reports the aberration polynomial coefficients resulting from operation of one (indicated as right-hand stack in the figure) or the other (left-hand) stack of diode bars. 

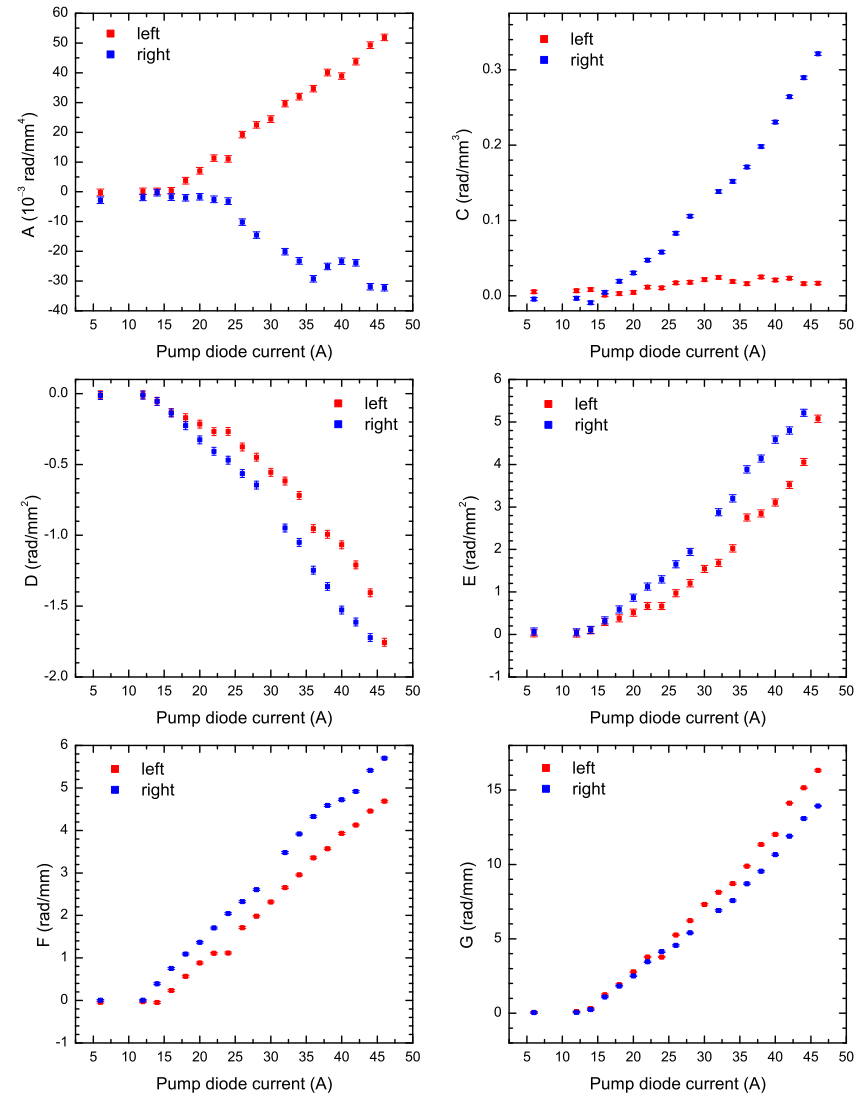

FIG. 8 Relevant aberration coefficients as a function of the pump diode current, in case of single-side pumped slab, by turning on one array (left or right) at time.

Figure 9 shows tilt and thermal lens data extracted from the polynomial coefficients as indicated in the case of symmetric pumping. It is worth noting that tilt always takes place in the same direction with a little difference in its amplitude related to the pumping asymmetry, indicating a source of symmetry breaking different from non-symmetrical thermal loading.

Interferometric measurements have been performed also during warm-up transients, acquiring fringe patterns at a maximum rate of $5 \mathrm{frame} / \mathrm{s}$ (the CCD exposure time was set at $7 \mathrm{~ms}$ ). Figure 10 shows the temporal evolution of the aberration polynomial coefficients in the first 2 minutes of diode operation.

Two different time scales are clearly visible. The first on is a time scale of 1-2 seconds in which the active slab absorbs pump radiation, expands and experiences a friction due to mechanical constraints. Consequently the slab sets in a new position because of these mechanical forces. On a longer time scale (40 to 60 seconds) heat diffusion towards the cooled large faces of the slab generates thermal gradients and stresses and causes slab deformation producing further optical aberrations. Interestingly from our dynamical measurements we can notice that $y$-tilt and coma are totally set in the first few seconds, and are thus produced by non uniform mechanical constraints. On the contrary $x$-tilt is almost totally due to thermal gradients. Defocusing, astigmatism and spherical aberration are mainly due to thermal gradients but show contributions from both sources of deformation. Comparing these results with Figure 2 we see that the optical resonant cavity sets up
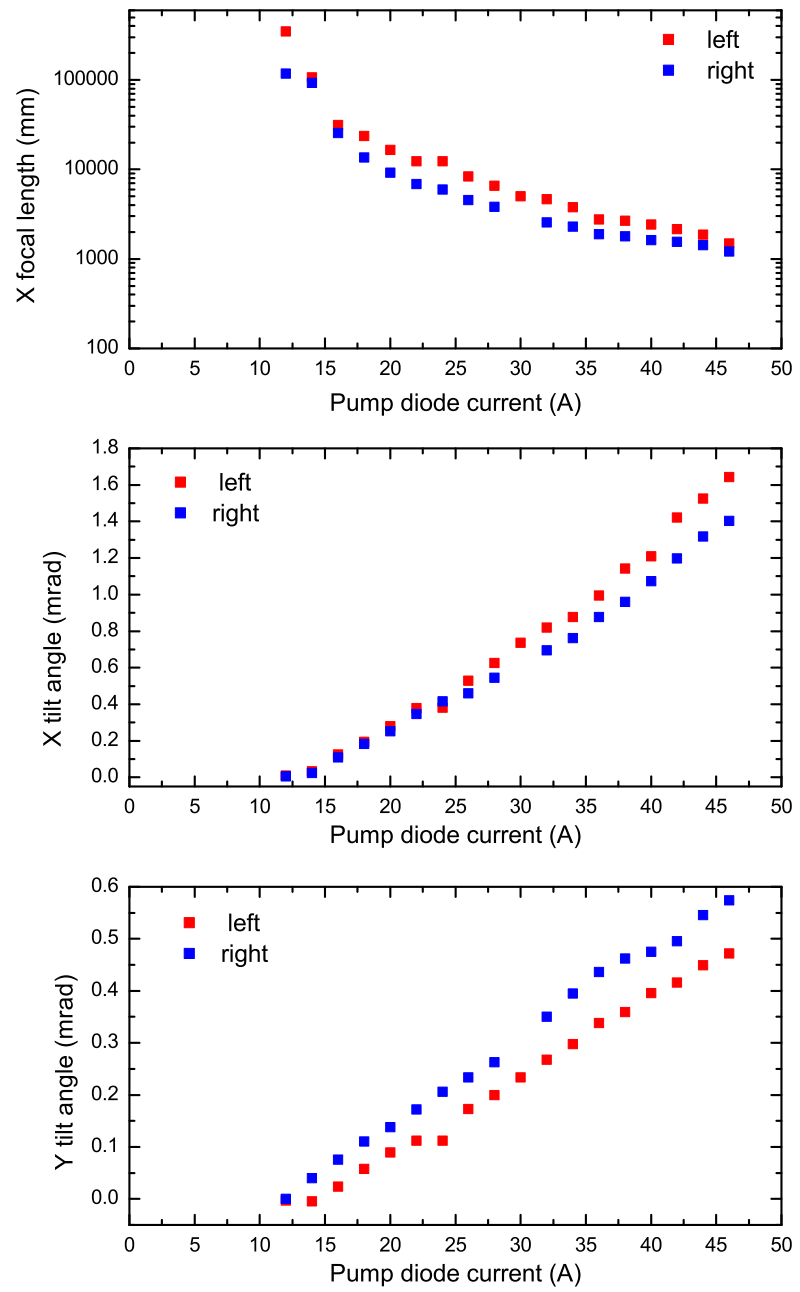

FIG. 9 Focal length in the $x$ direction and tilt angle in both transverse directions, as a function of pump diode current in case of single-side pumped slab as in Figure 8

after the first time scale deformations and is almost destroyed by the thermal deformations in the case of higher pump loadings. In the following section we will show FEM Computer simulations on a mechanical model of our system that show results consistent with these experimental observations.

\section{FEM COMPUTER-ANALYSIS OF THE CERAMIC SLAB DEFORMATIONS}

In order to study the effect of high thermal optical loading on the ceramic slab, we performed FEM numerical simulations. Simulations were made using the commercial bundle COSMOS-M which initially solves the thermal problem and then combines the thermal solution as a load together with mechanical boundary conditions to obtain deformations and stresses. The thermo-mechanical coefficients adopted in the present simulations are those of Ref. [1]. The geometrical model of the slab is depicted in Figure 11.

The active medium is uniformly pumped in the red-dashed region with radiation propagating along the $x$ axis and impinging on the two $18 \mathrm{~mm} \times 10 \mathrm{~mm}$ portions of the lateral faces. The pumping power is assumed to decay exponentially 

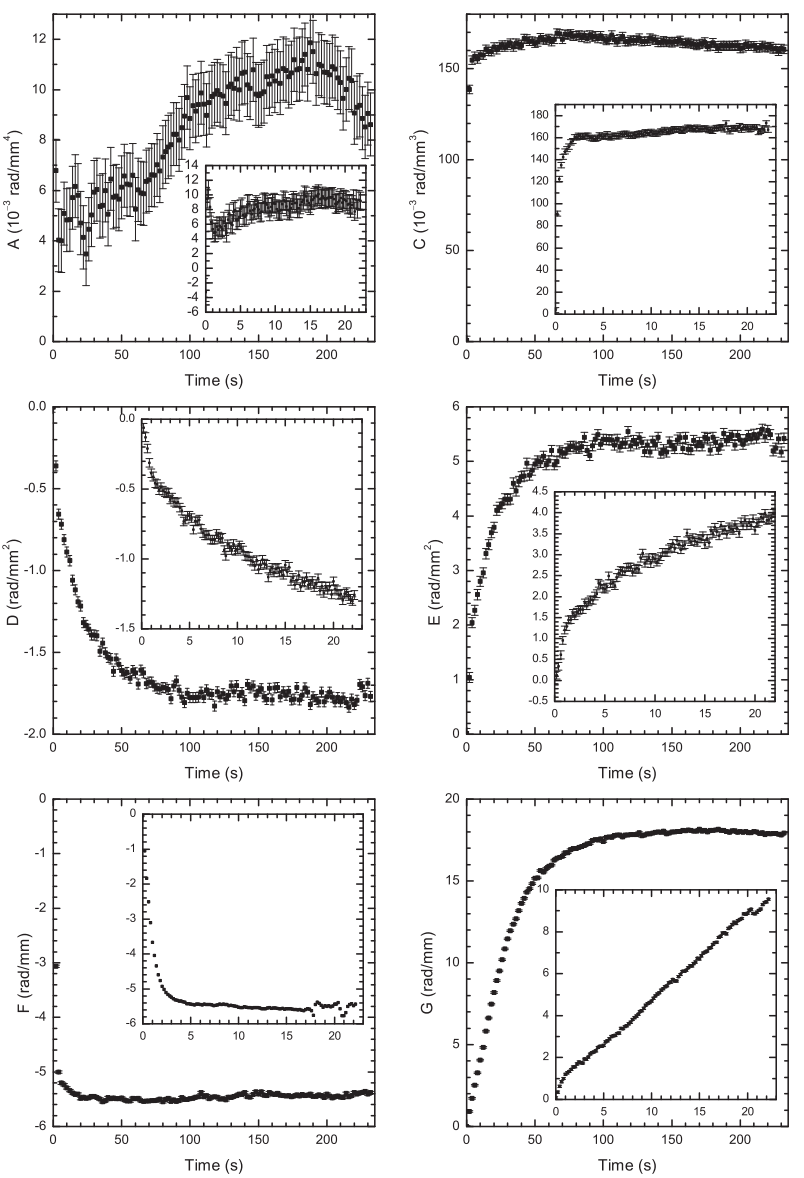

FIG. 10 Time evolution of the relevant aberration coefficients for symmetric pumping at $45 \mathrm{~A}$ of diode current: $t=0$ corresponds to diode laser turning-on. The insets show a more detailed view of the first $\sim 20 \mathrm{~s}$, emphasizing the occurrence of two different time scales.

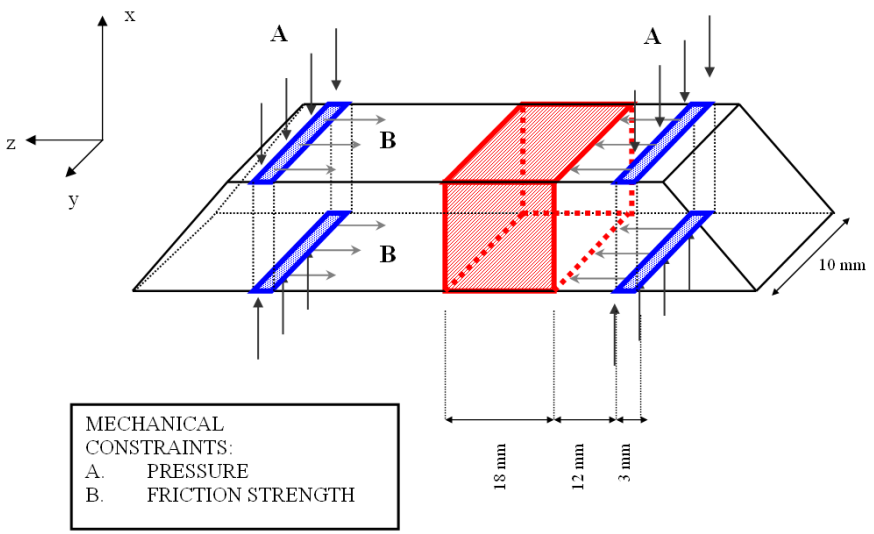

FIG. 11 Schematic view (not in scale) of the geometry adopted in the FEM simulations of the slab problem.

in the $x$ direction (the $3 \mathrm{~mm}$ thin dimension of the slab). To account for the emission spectrum bandwidth of our diode stacks, we assume the absorption coefficient to have a value in the interval $3-5 \mathrm{~cm}^{-1}$. This corresponds to an average absorption on a spectral region of $6 \mathrm{~nm}$ around the $808 \mathrm{~nm}$ peak of a $1 \%$ doped Nd:YAG. Changes in the doped YAG absorption coefficient naturally affect the thermal load distribution over different slices of the slab volume. In our modeling the pumped surfaces are set at a fixed temperature of $20^{\circ} \mathrm{C}$, as they are, in the experiment, conductively cooled by a flowing liquid from an external chiller.

The thermal loading caused by the absorbed laser diodes' radiation is varied between 150 and $450 \mathrm{~W}$ per stack. In facts, even if we irradiate our slab with $500 \mathrm{~W}$ from each stack, only a fraction of this power (around 90\%) is absorbed by the slab and part of this absorbed power is lost through non-radiative mechanisms. Typically in a non-lasing system based on a $1 \%$ doping Nd:YAG active medium a fraction around $40 \%$ decays through thermal channels [11]. Nevertheless our system encloses the pumping units, facing the slab, in a metallic chamber, so that a significant part of the power lost through radiative decays is eventually coupled back onto the slab volume producing further heat. For this reason we have tested thermal models with a high percentage of power translated into thermal loading. For ease of schematic modeling this power is either coupled entirely onto the pumped fraction of the volume with the exponential decay function or divided into this exponential decay load and a uniform second load on the slab volume region included between the mechanical constraints. The mechanical constraints consist in 4 rubber stripes, representing the water cooling chamber gaskets, subject to a normal pressure. These stripes obviously produce a friction and do not allow the slab to freely expand in its larger elongation direction $(z)$, when subject to the pump radiation absorption heating. This mechanical resistance is in real facts not uniform over the entire mechanical constraints and this causes the slab to tilt or bend as shown in two cases by the 3D deformation plots of Figures 12 and 13.

In our analysis we simulated a different friction between the gaskets and the slab surfaces by setting a different value of the elasticity modulus $E$ for each gasket section as detailed in the table. We have tested several different parameter sets and the most representative are summarized in Tables 1 and 2.

\begin{tabular}{|l|l|l|l|l|l|}
\hline Case & $\begin{array}{l}\text { Aborption } \\
\text { coefficient } \\
{\left[\mathrm{cm}^{-1}\right]}\end{array}$ & Pressure & $E_{\text {up }}$ & $E_{\text {down }}$ & $\begin{array}{l}\text { Thermal } \\
\text { Load } \\
{\left[\mathrm{kg} \mathrm{cm}{ }^{-2}\right]}\end{array}$ \\
{$\left[\mathrm{N} \mathrm{m}^{-2}\right]$} & {$\left[\mathrm{N} \mathrm{m}^{-2}\right]$} \\
\hline Exp. & $3-5$ & $500-1500$ & n.a. & n.a. & $150-450$ \\
1 & 4 & 1500 & $1 \times 10^{9}$ & $1 \times 10^{8}$ & 150 \\
2 & 3 & 1500 & $1 \times 10^{9}$ & $1 \times 10^{8}$ & 150 \\
3 & 3 & 1500 & $1 \times 10^{9}$ & $1 \times 10^{8}$ & 300 \\
4 & 3 & 1500 & $1 \times 10^{9}$ & $1 \times 10^{7}$ & 300 \\
5 & 3 & 1500 & $1 \times 10^{9}$ & $1 \times 10^{7}$ & 450 \\
6 & 4 & 1500 & $1 \times 10^{9}$ & $1 \times 10^{7}$ & 450 \\
7 & 4 & 1500 & $1 \times 10^{9}$ & $1 \times 10^{7}$ & $150+300$ \\
8 & 4 & 500 & $1 \times 10^{9}$ & $1 \times 10^{7}$ & $150+300$ \\
\hline
\end{tabular}

TABLE 1 Comparison of the resulting beam-tilt data obtained in our numeric simulation and from the interferometric tests. UD=symmetric loading, $U=$ heat loading from the top of the slab, $D=$ heat loading from the bottom of the slab. The last column indicates the ratio between the effect of mechanical and thermal asymmetry. (n.a.: not available) 


\begin{tabular}{|l|l|l|l|l|}
\hline Case & $T_{U D}$ & $\begin{array}{l}T_{U} \\
{[\mathrm{mrad}]}\end{array}$ & $\begin{array}{l}T_{D} \\
{[\mathrm{mrad}]}\end{array}$ & $\begin{array}{l}r \\
{[\mathrm{mrad}]}\end{array}$ \\
\hline Exp. & 2.3 & 1.4 & 1.6 & 7.5 \\
1 & 0.25 & 0.07 & 0.21 & 1.0 \\
2 & 0.22 & 0.08 & 0.17 & 1.4 \\
3 & 0.41 & 0.13 & 0.31 & 1.2 \\
4 & 0.46 & 0.16 & 0.34 & 1.4 \\
5 & 0.67 & 0.18 & 0.50 & 2.1 \\
6 & 0.77 & 0.20 & 0.62 & 1.8 \\
7 & 0.99 & 0.60 & 0.46 & 7.1 \\
8 & 0.98 & 0.58 & 0.44 & 7.5 \\
\hline
\end{tabular}

TABLE 2 Comparison of the resulting beam-tilt data obtained in our numeric simulation and from the interferometric tests. UD=symmetric loading, $U=$ heat loading from the top of the slab, $D=$ heat loading from the bottom of the slab. The last column indicates the ratio between the effect of mechanical and thermal asymmetry. (n.a.: not available)

For the sake of simplicity in defining the geometrical model for the FEM analysis package, we have disposed the slab with its $x$-axis vertical. As a consequence the diode stacks light the slab from above and below in the numerical simulations (corresponding to left and right in the experiments).
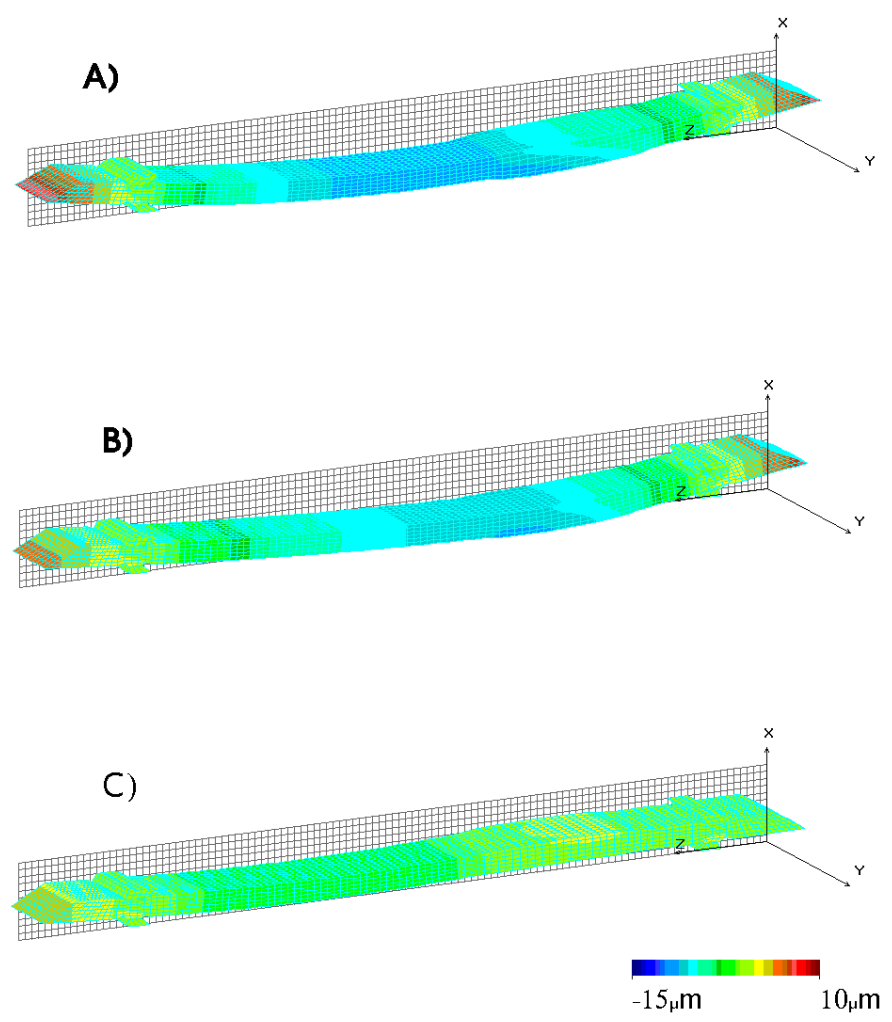

FIG. 12 Geometrical deformations of the slab obtained from the thermo-mechanical computer simulations relative to parameters of case 6 of Table I. A) Slab pumped from both sides (Up and Down). B) Slab pumped from the upper side. C) Slab pumped from the lower side.

The purpose of the present work is that of finding the thermomechanical calculation parameters that quantitatively reproduce the beam tilt measured in our experimental system. For this reason, in our simulations, we tested the same situations experimentally investigated, namely those produced either
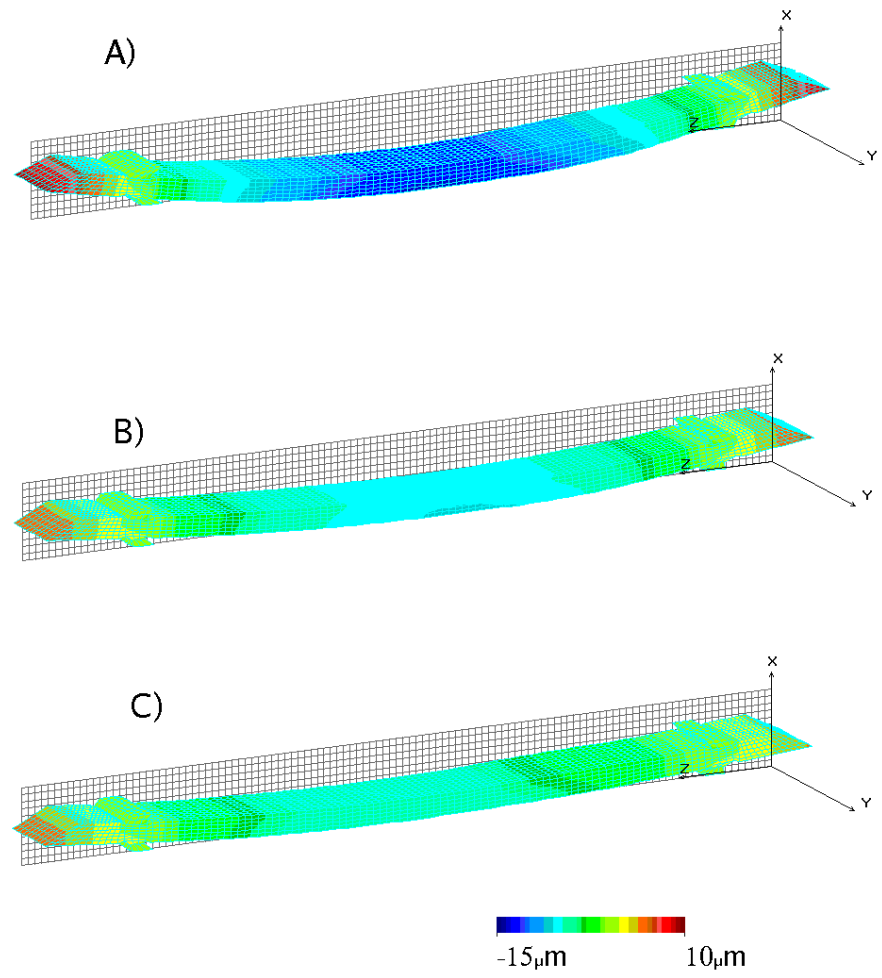

FIG. 13 Geometrical deformations of the slab obtained from the thermo-mechanical computer simulations relative to parameters of case 8 of Table I. A) Slab pumped from both sides. B) Slab pumped from the upper side. C) Slab pumped from the lower side.

turning both diodes on, or lighting one diode at a time. The tilt values reported in the colums 2, 3 and 4 of Table 2 are the tilt of a plane wavefront zig-zag propagating through the slab in the case of symmetric (up and down) and non-symmetric (up or down at a time) pumping respectively. The transmitted wavefront tilt is calculated as being the double of the tilt produced between the entrance and the exit faces of the slab. A more precise calculation based on the exact surfaces obtained in this analysis and on a ray-tracing method will be discussed elsewhere. In order to better compare the numerical and experimental tilts we define an evaluation parameter $r$ as the ratio between the tilt difference for asymmetrical thermal load and the tilt produced in case of symmetrical pumping:

$$
r=\frac{T_{D}-T_{U}}{T_{U D}}
$$

where $T_{D}\left(T_{U}\right)$ is the tilt produced on the beam operating the stack below (above) the slab and $T_{U D}$ is the tilt produced operating both the two stacks. In all the simulations reported in Table I an asymmetry is introduced between the elasticity modulus of the rubber stripes above the slab $\left(E_{\text {up }}\right)$ and below the slab $\left(E_{\text {down }}\right)$. The results reported in Tables 1 and 2 show that a beam tilt quantitatively comparable with the experiment and with the correct proportion of bending produced by thermal and mechanical asymmetry is obtained in our case study number 8 . In this modeling $150 \mathrm{~W}$ of optical power from the diode arrays are converted into heat directly from the first absorption, while $300 \mathrm{~W}$ are uniformly re-absorbed in a volume $10 \mathrm{~mm} \times 3 \mathrm{~mm} \times 70 \mathrm{~mm}$ after emission from the slab (fluorescence). 
The geometrical deformations obtained from our numerical model are shown in the three dimensional plots of Figure 12 for the parameters of case study number 6 and in Figures 13 for the parameters of case study number 8 . Case 6 most clearly shows the effect of a non-symmetric heating, while case 8 is the one producing the best ratio $r$. Introducing a difference in the elasticity modulus of the two rubber stripes situated on the same side of the slab we can add a rigid tilt of the slab as a consequence of thermal expansion.

\section{LASER CAVITY STABILITY DISCUSSION AND CONCLUSIONS}

Making use of a simple calculation [12] of the cavity $g$ parameters corrected to account for the interferometrically measured thermal lens, we can state that our laser emission is not destroyed by thermal lens effects. Indeed the cavity in the present design is brought deeper into the stability region of the $g_{1}-g_{2}$ parameter-space, by the relatively small pump induced thermal lens. On the contrary our measurements and numerical simulations indicate that the thermal induced slab bending produces an intracavity beam tilt of the order of 1 to $2 \mathrm{mrad}$. This beam tilt introduces a beam aperture reduction due to slab aperture coupling in the slab-mirror-slab path that can be extimated in the order of $12 \%$ from a simple geometric calculation. This aperture reduction produces a worst beam to active medium coupling and an extra cavity loss contribution that can naturally account for the extracted power reduction at higher thermal loading regimes. The comparison of our numerical simulations results with the measured beam tilt in different pumping geometries enable us to obtain some important parameters that are greatly useful in the design and optimization procedures for the attainment of stable high power $\mathrm{CW}$ regime systems. Such parameters include an average pump absorption coefficient or the percentage of pump power directly converted into heat.

\section{ACKNOWLEDGMENTS}

The authors thank Pietro Ferraro for useful discussion and suggestions. This work was performed within the National Project LaCer (Laser Ceramico) fundend by the Italian Ministery for University and Research (M.U.R.) in the framework of a F.A.R. action.

\section{References}

[1] M. Ciofini, A. Lapucci, “Compact scalable diode-pumped Nd:YAG ceramic slab laser" Appl. Optics 47, 6174-6179 (2004).

[2] A. Lapucci, M. Ciofini, "Efficiency optimization for a diode pumped Nd:YAG ceramic slab laser" Appl. Optics 44, 4388-4393 (2005).

[3] M. Ciofini, E. Favilla, A. Lapucci, E. Sani, “Propagation Parameters of the beam extracted from a diode pumped Nd:YAG ceramic slab laser with a hybrid stable-unstable resonator", Journal of optics and Laser Technology (2006),doi;10.1016/j.optlastec.2006.10.012 (available on line).

[4] I.Shoji, T.Taira, A.Ikesue, "Thermally-induced-birefringence effects of highly $\mathrm{Nd}^{3+}$-doped $\mathrm{Y}_{3} \mathrm{Al}_{5} \mathrm{O}_{12}$ ceramic lasers", Opt. Mater. (2006), doi:10.1016/J.optmat.2005.12.012.

[5] B. Neuenschwander, R. Weber, H. P. Weber, "Determination of the thermal lens in solid-state lasers with stable cavities", IEEE J. Quantum Elect. QE-31 6, 1082-1087 (1995).

[6] V. S. Ermolaev, S. B. Biryuchinskii, M. V.Inochkin, A. I.Pavlishin, "Study of the thermal lens in a $150 \mathrm{~W}$ solid-state processing laser with semiconductor pumping" J. Opt. Technol. 70, 789-793 (2003).

[7] H. Yoshida, M.Nakatsuka, N. Takeuchi, H.Okada, H.Fujita, “Thermal lens correction of Nd:YAG rod laser used with thermo-optical compensation element of solid material" Tech. Dig. CLEO/Europe 2005, p.73 (2005).

[8] W. A. Clarkson, N. S. Felgate, D. C. Hanna, "Simple method for reducing the depolarization loss resulting from thermally induced birefringence in solid-state lasers" 0pt. Lett. 24, 820-822 (1999).

[9] S. Biswal, J. Nees, G. Mourou, "A simple technique to remove thermal distortions in pulsed solid-state lasers" in OSA Trends in Optics and Photonics, C. Pollock and W. Bosenberg eds., Vol.10 paper HP 9 (OSA, 1997).

[10] see for example: D.Malacara Optical Shop testing (Wiley, New York, 1992).

[11] I. Shoji, T. Taira, A. Ikesue, K. Yoshida, "Reduction of the thermal load in highly $\mathrm{Nd}^{3+}$-doped ceramic YAG by laser oscillation" paper MB1, in Advanced Solid-State Photonics (Tech.Digest, OSA, 2004).

[12] H. P. Kortz, R. Ifflaender, H. Weber, "Stability and beam divergence of multimode lasers with internal variable lenses" Appl. 0ptics 20, 4124-4134 (1981). 